\title{
Experimental investigation of intermittent airflow separation and microscale wave breaking in wavy two-phase pipe flow
}

\author{
P. Vollestad ${ }^{1, *}$, A.A. Ayati ${ }^{1}$, A. Jensen ${ }^{1}$ \\ 1 Department of Mathematics, University of Oslo, 0316 Oslo, Norway \\ *Corresponding author: pettervo@math.uio.no, +4748147276
}

\begin{abstract}
We perform an experimental analysis of co-current, stratified wavy pipe flow, with the aim to investigate the effect of small scale wave breaking (microscale breaking) on the airflow. Particle image velocimetry (PIV) is applied simultaneously in the gas and liquid phase. Active wave breaking is identified by high levels of vorticity on the leeward side of individual waves, and statistics of the airflow above breaking and non-breaking waves are extracted from the gas-phase velocity fields.

Keeping the liquid superficial velocity constant $\left(U_{s l}=0.1 \mathrm{~m} / \mathrm{s}\right)$, we consider two experimental cases of different gas flow rates. The lowest flow rate $\left(U_{s g}=1.85 \mathrm{~m} / \mathrm{s}\right)$ is slightly higher than the onset of microscale breaking, while the higher flow rate $\left(U_{s g}=2.20 \mathrm{~m} / \mathrm{s}\right)$ is within the regime where wave breaking is observed to be frequent, and the rms interface elevation $\eta_{r m s}$ is independent of gas flow rate.

Results show that for the lowest gas flow rate considered, active wave breaking has a stabilizing effect on the airflow above the waves, reducing the sheltered region on the leeward side of the wave and the turbulence above the wave crest compared with non-breaking waves at similar steepness. At the higher gas flow rate the effect of active wave breaking is found to be small, and the main geometrical properties of the waves are found to dominate the evolution of the separated flow region.
\end{abstract}

\section{Introduction}

Airflow over water waves represents a challenging problem relevant both in nature and for engineering applications, such as stratified two-phase pipe flows, which is the focus of this work. Considering wind over waves, two of the most characteristic features for the liquid and gas phase respectively is wave breaking and airflow separation. Wave breaking occurs when fluid elements at the surface overtakes the general waveform, resulting in increased turbulence in the liquid phase and a transfer of momentum and energy from the waves to the underlying flow (Jessup et al., 1997; Banner \& Melville, 1976). Airflow separation over surface waves is characterized by increased turbulence in the air phase as the shear layer separates from the interface, and a significant displacement of the streamlines relative to the wave shape. This results in a distribution of pressure and shear forces along the wave profile which deviate significantly from the non-separated case (Weissman, 1986). Analysis of these phenomena in the pipeflow literature are scarce, hence we refer to the air-sea literature for a background to the understanding of wave breaking and airflow separation.

Banner \& Melville (1976) and Gent \& Taylor (1977) considered separation above water-waves as "the occurrence, in a frame of reference moving with the waves, of a streamline leaving the water surface". This requires the surface velocity of the interface to match the wave speed, which is the criterion for incipient wave breaking. Hence, it was concluded that airflow separation may only occur in conjunction with wave breaking. This criterion has been applied by researchers modelling drag on the sea surface (Kudryavtsev \& Makin, 2001). However, early flow visualization techniques indicated 
that airflow patterns resembling the separated flow over solid obstacles may occur over non-breaking waves (Kawai, 1981, 1982; Weissman, 1986). According to Weissman (1986), the separation criterion utilized by Gent \& Taylor (1977) and Banner \& Melville (1976) is not appropriate when the surface is moving, as airflow separation over moving boundaries may have stagnation points away from the wall. As described by Weissman (1986); "the "separation" we are concerned with is that of the shear layer closest to the surface...". Buckley \& Veron (2016) considered the airflow as separating "if the near-surface, high vorticity layer characteristic of an attached boundary layer is ejected away from the water surface and the surface vorticity is near zero or negative". A similar criterion was also applied by Reul et al. (2008) and Sullivan et al. (2018b).

Applying the definition of (shear layer) separation by Weissman (1986); Buckley \& Veron (2016); Reul et al. (2008), it has been documented both experimentally (Veron et al., 2007; Tian et al., 2010) and numerically (Sullivan et al., 2018b) that separation may occur over non-breaking waves. Wave breaking has been found to be a sufficient, but not a necessary condition for airflow separation (Tian et al., 2010; Sullivan et al., 2018b).

Wave breaking is separated into categories related to the severity and visual characteristics of the breaking process. Babanin (2011) divides wave breaking into plunging, spilling and microbreaking. Microbreaking, or microscale breaking is a weak form of wave breaking, where surface tension prevents the jet formation and air entrainment into the liquid phase as the wave breaks (Tulin \& Landrini, 2001; Jessup et al., 1997; Siddiqui \& Loewen, 2006; Vollestad et al., 2019b). Microscale breaking waves are short (wavelength in the order of a few decimeters), wind generated waves with amplitudes up to a few centimeters. In the present work we focus our attention on these small scale breaking waves.

We consider stratified wavy two-phase pipe flow. These flows have traditionally been modelelled using a simple two-fluid model, which in addition to modified single phase correlation for the wall friction relies on a parametrization of the interface friction factor (Ullmann \& Brauner, 2006). The interfacial friction factor has typically been established through correlation studies based on 45 pressure drop and liquid hold-up measurements (see e.g. Tzotzi \& Andritsos (2013)), yielding little physical insight into the mechanisms that govern the momentum exchange at the interface. Recently, stratified gas-liquid pipe flow has been analyzed using PIV (particle image velocimetry) in the centerplane of the gas and liquid phase (Ayati et al., 2014, 2016; Birvalski et al., 2015, 2016). These studies have provided spatio-temporally and phase-averaged flow fields, and hence valuable experimental data for model validation of more complex numerical models, and new insight into the dynamics of the two phases. Previous analysis of the experimental setup considered in this work (Vollestad et al., 2019a) has shown that intermittent airflow separation is a frequent event as the gas flow rate in increased into the amplitude saturation regime described by Ayati et al. (2015), and microscale wave breaking has been observed when the superficial gas velocity $U_{s g} \geq 1.8 \mathrm{~m} / \mathrm{s}$, phase $k$ is $U_{s k}=\dot{m}_{k} /\left(\rho_{k} A\right)$, where $\dot{m}_{k}$ and $\rho_{k}$ is the mass flow rate and density of phase $k$, while $A$ is the cross sectional area of the pipe). Microscale wave breaking was found to influence the liquid phase down to a depth of approximately $1 \mathrm{~cm}$ below the crest (approximately one significant wave height), increasing the turbulent dissipation rate below the crest by a factor 3-4 compared with non-breaking waves (Vollestad et al., 2019b). These results are consistent with observations of microscale breaking waves in wind-wave tanks (Siddiqui \& Loewen, 2007, 2010), indicating that the main features of microscale breaking waves in two-phase pipe flow are similar to the processes occurring in the ocean.

When small scale wind waves go from incipient to active breaking, a thin spilling region is generated on the leeward side of the wave crest, resulting in increased turbulence and vorticity in the liquid phase. The present understanding of how active breaking influences the airflow field above waves and airflow separation is however far from complete (Sullivan et al., 2018b). While previous 
analysis of two-phase pipe flow has documented that both airflow separation and microscale wave breaking occurs for sufficiently high gas flow rates, the link between the two has not previously been investigated in a pipe flow setup.

Analyzing airflow over waves by PIV in a rectangular wind-wave tank, Reul et al. (2008) found that the separation induced by breaking waves is stronger and more consistent than separation over non-breaking waves. Reul et al. (2008) performed measurements over waves ranging from microbreaking to plunging, the claim is not specific for microscale breaking waves. Banner (1990) evaluated the pressure and velocity field over small scale incipient and actively breaking waves, using a slope exceedance threshold to distinguish between breaking and non-breaking waves. Banner (1990) reports that while there is a significant phase shift of the pressure field also for unbroken steep waves, active wave breaking results in an increased (order $100 \%$ ) phase shift and form drag above the wave profiles. Similar results were found by numerical analysis by Maat \& Makin (1992), who concluded that the increased local roughness in the spilling region enhanced the asymmetrical surface pressure pattern. Recently, Sullivan et al. (2018b) performed LES simulations of airflow above wave profiles taken from the experiments of Banner (1990). Example wave profiles of incipient and actively breaking waves $\left(a k=0.25,0.28, c / u_{*}=1.58,1.23\right.$ for incipient and active breaking respectively) were investigated. Based on parameter tuning of the numerical model they found that the wave steepness, not the enhanced surface drift caused by wave breaking, was the critical property inducing large form drag over the actively breaking waves, indicating that the geometrical properties of the waves (and not the state of wave breaking) dictates the surface drag. Recent two-fluid DNS simulations by Yang et al. (2018) has shown that while a plunging breaker will significantly impact the airflow field, a spilling breaker (initial steepness $a k=0.35$ ) under relatively strong wind forcing $\left(c / u_{*}=3.7\right)$ does not significantly influence the airflow as the wave transitions from a smooth unbroken wave form to a spilling breaker. Simulations by Yang et al. (2018) indicates that the form drag remains essentially constant as the wave evolves into an active spilling breaker.

In this work we perform simultaneous two-phase PIV, to obtain $2 \mathrm{D}$ velocity fields in both the gas and liquid phase. The technique was first applied by Ayati et al. (2014) for two-phase pipe flow. The novelty of this work lies in the simultaneous assessment of active wave breaking and evaluation of the airflow above individual waves. Wave breaking is assessed by the method of Vollestad et al. $(2019 b)$, evaluating the rms vorticity in the liquid phase on the leeward side of the crest. Statistics of the flow field over breaking and non-breaking waves at two different gas flow rates are examined. The goal is to investigate how active wave breaking affects the airflow above waves compared with non-breaking waves of similar steepness. Understanding the effect of small scale wave breaking and its link to airflow separation in stratified two-phase pipe flow is assessed to be an important step in quantifying the physical processes responsible for the momentum exchange at the interface between the two phases. While the closed pipe geometry impacts the flow, making a direct comparison with the processes occurring in the ocean inaccurate, the results obtained in the present work may also provide insight into the processes occurring in an open system, as the underlying mechanisms (wave breaking and airflow separation) are the same. The simultaneous assessment of active wave breaking and airflow separation above microscale breaking waves has to the authors knowledge not previously been documented for either an open or closed air-water system.

This paper is organized in six sections (including this introduction). In section 2 the experimental setup is presented. In section 3 the experimental cases and methodology are presented. Results are presented in section 4. Instantaneous flow fields are presented in section 4.1, while statistics of the airflow field above breaking and non-breaking waves are presented in section 4.2. A detailed analysis of waves within a narrow steepness band is performed in section 4.3. Finally, a discussion and concluding remarks are presented in section 5 and 6 . 


\section{Experimental setup}

The experiments were conducted at the Hydrodynamics laboratory, at the University of Oslo. The experimental setup consists of a 31 meter long, horizontal, transparent, acrylic pipe with an internal diameter $D=10 \mathrm{~cm}$. An overview of the experimental setup is presented in figure 1 . Air and water was supplied at the inlet by a frequency regulated fan and pump respectively. Here, honeycomb flow straighteners were placed to dampen turbulent fluctuations introduced at the inlet. The gas and liquid flow rates were measured using an Emerson MicroMotion Coriolis flow meter and an Endress Hauser Promass coriolis flow meter respectively. At the pipe outlet the fluids were discharged to a tank (at atmospheric pressure), and recirculated. The differential pressure in the air phase was measured over a 12.4 meter section, using a smar LD301 differential pressure gauge.

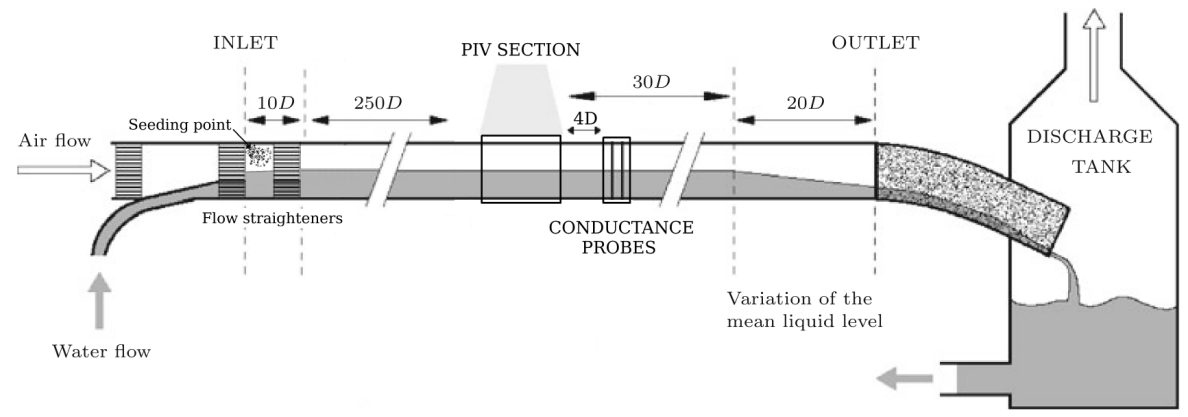

Figure 1: Schematic view of experimental setup.

A PIV section was placed $260 \mathrm{D}$ from the pipe inlet. Here, an optical correction box was placed around the pipe. The box was half filled with water to reduce optical distortion of the liquid phase PIV camera. A non-linear coordinate transform was obtained for each of the cameras applied by imaging a coordinate system placed in the pipe centerline, using the coordinate system described in Ayati et al. (2015). PIV was conducted simultaneously in the centerplane of both phases. A 147 mJ ND:YAG laser (532 nm wavelength light) illuminated the centerplane of the PIV section from above. The head of the laser optics was placed $50 \mathrm{~cm}$ above the pipe centerline, creating a thin $(<$ $1 \mathrm{~mm}$ ) laser sheet within the measurement section.

Three cameras were employed in the study. Their position and field of view (FOV) is shown in figure 2 .

- Liquid phase PIV camera. PCO.4000 camera with 4008x2672 pixels. Fitted with a $100 \mathrm{~mm}$ lens and a 1.6x teleconverter. The camera is directed upwards at an angle of $12^{\circ}$.

- Gas phase PIV camera. PCO.4000 camera with 4008x2672 pixels. Fitted with a $100 \mathrm{~mm}$ lens. The camera is directed downwards at an angle of $24^{\circ}$. A high downwards looking angle was necessary as the crescent shape of the waves meant that the pipe wall was repeatedly wetted well above the mean water level.

- Large field of view (LFV) camera. A Nikon D7200 with 6400x4000 pixels. Fitted with a 28 $\mathrm{mm}$ lens. The camera was directed downwards at an angle of $30^{\circ}$, and looking upstream at an angle of approximately $31^{\circ}$.

Due to the relatively high downward/upwards looking angle of the gas/liquid phase PIV cameras, these were fitted with Schleimpflug adapters, allowing the entire center plane of the pipe to be in focus. 


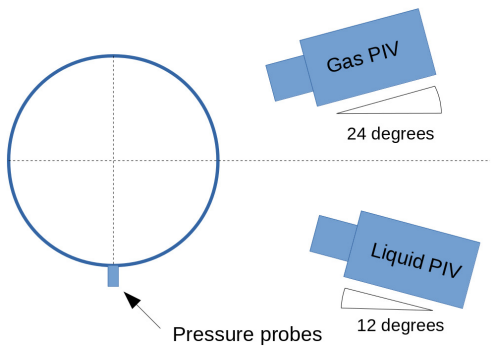

a)

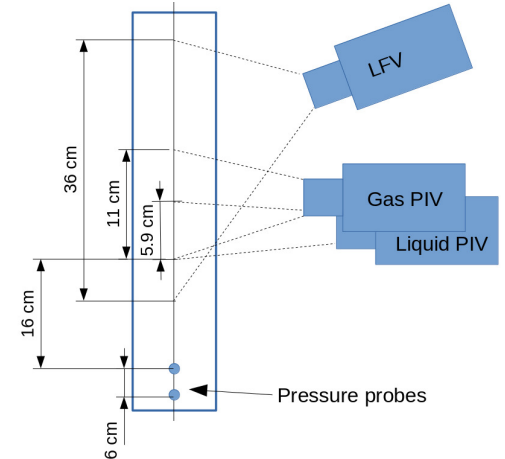

b)

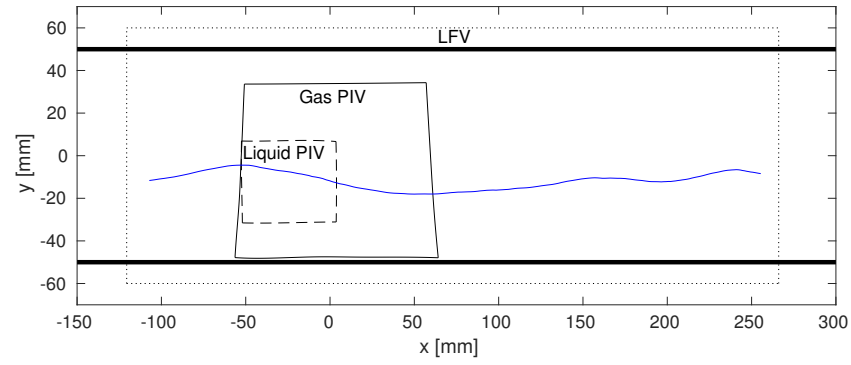

c)

Figure 2: Schematic view of PIV measurement section. a) Looking downstream. b) Seen from above. c) FOV of all three cameras. Blue line represents an example interface profile (waves propagating to the right).

The gas phase was seeded with small water particles, generated by a high-pressure atomizer. The particles were injected into the gas-phase at the inlet. According to the manufacturer, $72 \%$ of the droplets are below $6 \mu \mathrm{m}$, and it has previously been shown by Ayati et al. (2014) that the particles exhibit a Stokes number well below 1 for the flow rates of interest.

Initial analysis using polyamide particles in the liquid phase showed that light reflections from particles near the spilling region of microscale breaking waves influenced the gas-phase PIV images, causing unreliable results near the gas-liquid interface. This issue was overcome by using fluorescent particles in the liquid phase and fitting the gas-phase PIV camera with a bandpass filter centered at $532 \mathrm{~nm}$ (midopt BP525 Light Green Bandpass Filter). The liquid phase was seeded with fluorescent particles made from a commercially available acrylic paint (Lefranc \& Bourgeois, Fluorescent light orange). Particles made from this paint were used also by Birvalski et al. (2015) to perform PIV in the liquid phase of two-phase pipe flow. The particles were extracted in a settling process, similar to the process described by Birvalski (2015). Visual inspection of the particles under the microscope revealed that the particle size typically ranged from 5 to $20 \mu \mathrm{m}$.

For efficient interface detection, Rhodamine $\mathrm{B}$ was added to the water at a concentration of $5 * 10^{-5} \mathrm{~g} / \mathrm{l}$. A filter closely matching the wavelength emitted from the rhodamine $(\mathrm{B}+\mathrm{W} 041$ red/orange filter) was added to the LFV camera. This removed the green signal from the droplets in the air-phase, allowing for efficient interface detection from the LFV camera. The interface was detected by a thresholding technique, similar to the technique applied by Vollestad et al. (2019b), and transferred to the gas and liquid phase PIV images by applying a cubic coordinate transform. By looking through many PIV images (gas and liquid) overlaid the detected interface, the accuracy of the interface detection and coordinate transform is assessed to be approximately $0.4 \mathrm{~mm}$, similar to the PIV resolution.

Two pressure probes (Kulite XTL-190) were mounted flush to the pipe bottom (position indicated in figure 2). The probes measured the pressure at a frequency of $2000 \mathrm{~Hz}$. The pressure was 
normalized by a normalization filter (output centered at 0 with standard deviation 1 ) based on the previous 30 seconds of data sampling. When a pressure peak (associated with a wave crest) above a threshold $\sigma$ was detected by the downstream pressure probe, a cross-correlation of the previous one second of data was performed between the two probes, to estimate the wave speed. This estimate was used to evaluate a trigger delay to the PIV system, allowing the leeward side of the wave to be repeatedly present within the liquid phase PIV FOV as the PIV images were acquired (necessary for the analysis performed in this work). The pressure probe trigger system is described in more detail in Vollestad et al. (2019b).

The two laser pulses were triggered with a $\Delta t$ of 100-120 $\mu \mathrm{s}$ (depending on the flow rate combinations applied). Note that while the PIV cameras acquired double images (one image per laser pulse), the LFV camera was set to trigger before the first laser pulse, and had an exposure time which was longer than the laser pulse $\Delta t$, i.e. the LFV image was double exposed. During the laser $\Delta t$, the waves would move less than $0.1 \mathrm{~mm}$, which is a negligible fraction of the overall wave shape, and less than the accuracy of the interface detection.

A conductance wave gauge was placed $4 \mathrm{D}$ downstream of the PIV section, measuring the interface elevation at $500 \mathrm{~Hz}$. This consists of two double-wire probes of $0.3 \mathrm{~mm}$ diameter separated by $4 \mathrm{~mm}$. The two probes were placed in the center of the pipe with a distance $6 \mathrm{~cm}$ in the streamwise direction. For more details on the wave-gauges the reader is referred to Ayati et al. (2015).

PIV was performed with a cascade of sub-pixel passes, with a final interrogation window of $52 \times 26$ pixels in the gas phase, and $32 \times 32$ pixels in the liquid phase, using $50 \%$ overlap. This translates to a spatial resolution of $0.75 \times 0.38 \mathrm{~mm}^{2}$ in the gas phase, and $0.24 \times 0.24 \mathrm{~mm}^{2}$ in the liquid phase. Spurious vectors were detected by standard methods based on a local median filtering (Raffel et al., 2018), and replaced by cubic interpolation.

\section{Methodology}

\subsection{Description of experimental cases}

We consider two experimental cases, where the liquid superficial velocity is kept constant at $U_{s l}$ $=0.1 \mathrm{~m} / \mathrm{s}$, while the gas superficial velocity $\left(U_{s g}\right)$ is increased from $1.85 \mathrm{~m} / \mathrm{s}$ to $2.20 \mathrm{~m} / \mathrm{s}$. These experimental cases were chosen as they appear in two different regimes in the flow map. As shown by Vollestad et al. $(2019 b)$, for this liquid flow rate the first interfacial waves are observed at $U_{s g}$ $=1.3 \mathrm{~m} / \mathrm{s} . \quad \eta_{r m s}$ (the rms of the interface elevation fluctuations) at the measurement section is observed to increase with $U_{s g}$ until $U_{s g} \approx 2.0 \mathrm{~m} / \mathrm{s}$. At this gas superficial velocity a regime of "amplitude saturation" is observed, and the main characteristics of the wave field are relatively independent of the gas flow rate. This implies that an equilibrium between increased wind input and dissipation is reached, which is reminiscent of the equilibrium range of the ocean wave spectrum (Phillips, 1958, 1985). Vollestad et al. (2019b) found that the first evidence of wave breaking was observed at $U_{s g}=1.8 \mathrm{~m} / \mathrm{s}$. Increasing the gas velocity into the amplitude saturation regime was observed to increase both the frequency and intensity of the wave breaking events. Hence, for both experimental cases considered we expect to observe both breaking and non-breaking waves at the measurement section.

In order to observe a reasonable number of both breaking and non-breaking waves (needed to obtain reliable statistics) the lowest $U_{s g}$ is set to $1.85 \mathrm{~m} / \mathrm{s}$ rather than $1.8 \mathrm{~m} / \mathrm{s}$. The trigger threshold $\sigma$ was set to 1.6 for the $U_{s g}=1.85 \mathrm{~m} / \mathrm{s}$ case and 1.4 for the $U_{s g}=2.20 \mathrm{~m} / \mathrm{s}$ case. The trigger threshold results in a bias for detecting larger amplitude waves in the system, as the smaller waves will not trigger the PIV system. Setting a higher trigger threshold for the $U_{s g}=1.85 \mathrm{~m} / \mathrm{s}$ case was necessary to observe a reasonable fraction of both breaking and non-breaking waves.

The main characteristics of the two experimental cases is presented in table 1 , while the spectra (evaluated by the wave probes) is presented in figure $3 . C_{p}$ is the characteristic wave speed evaluated 
by cross-correlation of the conductance wave probes, $\lambda_{p}$ is the peak wavelength, evaluated at the peak of the spectrum, and $\bar{h}$ is the mean water level in the center of the pipe, evaluated by the wave probes. This is used to evaluate the bulk gas and liquid velocities $\left(U_{b g} / U_{b l}\right)$. The relationship between the superficial velocity of phase $k$ and the bulk velocity is $U_{s k}=A_{k} U_{b k} / A$ where $A$ and $A_{k}$ is the pipe cross-sectional area and the area occupied by phase $k . \quad \lambda_{p}$ and $\bar{h}$ implies that $k_{p} h \approx 1.3-1$ for the two cases considered, and the waves can be classified as waves at intermediate depth. The wave age $C_{p} / u_{*}$ indicates that the waves are young, wind-driven waves. The interfacial friction velocity $u_{*}$ was estimated using a force balance over a 12.4 meter section of the pipe. Here the average pressure drop in the air phase was measured, and the wall friction was estimated by the Colebrook-White formulation. Subtracting the wall friction forces from the measured pressure drop yields an estimate of the interface friction $\tau_{i}$, and the friction velocity is then evaluated as $u_{*}=\sqrt{\tau_{i} / \rho}$. Note that this is a global estimate of the wave age, and as the wave field develops along the pipe, deviations from the values listed in table 1 may occur locally. Further details on the development of the two-phase flow field with varying $U_{s g}$ has been discussed by Ayati et al. (2015) and Vollestad et al. (2019b).

Table 1: Overview of experimental cases. $N_{P I V}$ is the number of PIV velocity fields analyzed for each case.

\begin{tabular}{|c|c|c|c|c|c|c|c|c|c|}
\hline $\begin{array}{c}U_{s l} \\
{[\mathrm{~m} / \mathrm{s}]}\end{array}$ & $\begin{array}{c}U_{s g} \\
{[\mathrm{~m} / \mathrm{s}]}\end{array}$ & $\begin{array}{c}\eta_{r m s} \\
{[\mathrm{~mm}]}\end{array}$ & $\begin{array}{c}\lambda_{p} \\
{[\mathrm{~mm}]}\end{array}$ & $\begin{array}{c}C_{p} \\
{[\mathrm{~m} / \mathrm{s}]}\end{array}$ & $\begin{array}{c}\bar{h} \\
{[\mathrm{~mm}]}\end{array}$ & $\begin{array}{c}U_{b l} \\
{[\mathrm{~m} / \mathrm{s}]}\end{array}$ & $\begin{array}{c}U_{b g} \\
{[\mathrm{~m} / \mathrm{s}]}\end{array}$ & $\begin{array}{c}C_{p} / u_{*} \\
{[-]}\end{array}$ & $\begin{array}{c}N_{P I V} \\
{[-]}\end{array}$ \\
\hline 0.1 & 1.85 & 2.6 & 203 & 0.75 & 40.4 & 0.27 & 2.95 & 2.49 & 2395 \\
\hline 0.1 & 2.20 & 3.4 & 230 & 0.82 & 38.2 & 0.30 & 3.21 & 2.12 & 2000 \\
\hline
\end{tabular}

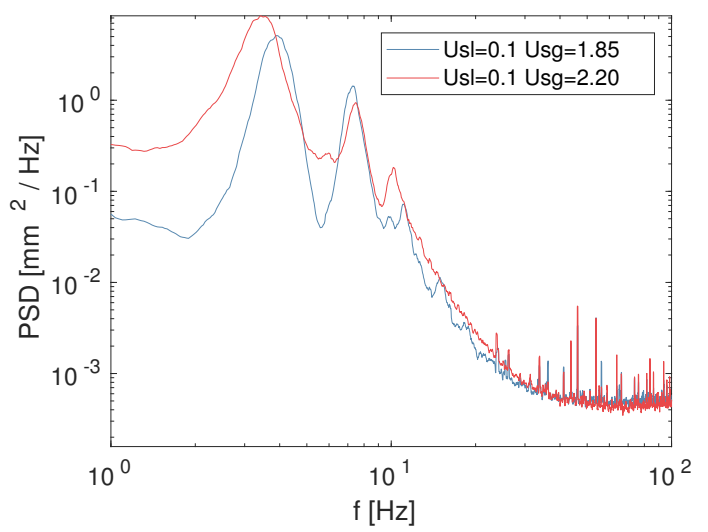

Figure 3: Frequency spectra from the waveprobes for the two experimental cases.

In the present work measurements are performed in the pipe center plane, and flow variations in the lateral direction are not quantified. As discussed by Vollestad et al. (2019b) the flow rates under investigation are within the "2D wave" regime defined by Tzotzi \& Andritsos (2013). The three-dimensionality of the wavefield (using the same two-phase flow loop) has previously been investigated by Strand (1993) and Smith et al. (2018). Strand (1993) used conductance wave probes at different crosswise planes of the pipe and found that for the flow rates under investigation in the present work, the normalized cross-correlation between the centerline and off-axis surface elevation exceeded 0.85 everywhere in the pipe. Smith et al. (2018) used x-ray tomography and found that the dominant wave components were well-described as $2 \mathrm{D}$ for the flow rates of interest. Near the pipe walls the waves were observed to have a crescent shape, and slightly larger wave amplitudes were observed compared with the pipe centerline. This is in line with visual observations of the highest flow rate case in the present work. Example images of breaking and non-breaking waves 
in the same two-phase pipe flow configuration presented by Vollestad et al. (2019b) show that the waves retain their $2 \mathrm{D}$ shape also during breaking.

\subsection{Regions of interest}

Two "regions of interest" (ROI's) are implemented to monitor the velocity field in the gas and liquid phase, and to identify characteristic features of wave breaking and airflow separation respectively. These are denoted GROI and LROI for the gas and liquid phase ROI. The ROI location relative to the wave profile is illustrated in figure 4 and described in more details in the following sections.

The local wave phase $\theta$ is used to define the relative location along the wave. $\theta$ is determined based on a zero-crossing procedure. The crest/trough was assigned a phase of $0^{\circ}$ and $180^{\circ}$ respectively, while the zero-down/zero-up crossings were assigned a phase of $90^{\circ}$ and $270^{\circ} /-90^{\circ}$. Phases in between were linearly distributed. This is illustrated in figure 4 .

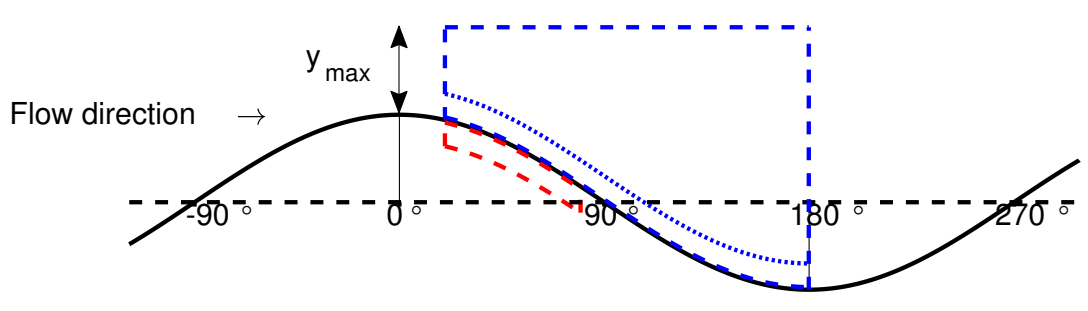

Figure 4: Illustration of the ROI's applied. Red dashed line: Liquid phase ROI to detect wave breaking. Blue dashed line: Gas phase ROI. Blue dotted line: Lower boundary of GROI applied when evaluating $\omega_{r m s, g}$

\subsubsection{Liquid phase ROI}

The LROI is defined for $20^{\circ}<\theta<80^{\circ}$, from the interface and $3 \mathrm{~mm}$ into the liquid phase. The velocity field within the liquid phase is used to classify waves as either breaking or non-breaking based on the criterion by Vollestad et al. (2019b). Vollestad et al. (2019b) monitored the rms vorticity (denoted $\omega_{r m s}$ ) within the same ROI. Waves with $\omega_{r m s}<60 \mathrm{~s}^{-1}$ were assessed to be nonbreaking, while waves with $\omega_{r m s}>100 \mathrm{~s}^{-1}$ were classified as active breaking. As we in the present study apply a spatial resolution closely matching the resolution used in Vollestad et al. (2019b), and hence resolve a similar fraction of the shear generated by wave breaking, the same criterion is used also here. The rms vorticity in the LROI is denoted $\omega_{r m s, l}$ in the remainder of this paper.

Only waves where the full LROI was present in the liquid phase PIV FOV are analyzed further. In table 2 the number of waves where the LROI was present in the liquid PIV FOV $\left(N_{L R O I}\right)$, as well as the number of breaking and non-breaking waves is presented. Note that waves with $60 s^{-1}<\omega_{r m s}<100 s^{-1}$ are classified as "intermediate", and not counted as either breaking or non-breaking. Further discussions on this methodology for distinguishing actively breaking and non-breaking waves is presented in Vollestad et al. $(2019 b)$.

Table 2: Number of waves found to be breaking/non-breaking in this study. $N_{L R O I}$ are number of waves with the full LROI present in the liquid PIV FOV.

\begin{tabular}{|c|c|c|c|c|c|}
\hline$U_{s l}$ & $U_{s g}$ & $N_{L R O I}$ & N breaking & N intermediate & N non-breaking \\
\hline 0.1 & 1.85 & 1670 & $452(27 \%)$ & $198(12 \%)$ & $1020(61 \%)$ \\
\hline 0.1 & 2.20 & 1426 & $571(40 \%)$ & $136(10 \%)$ & $719(50 \%)$ \\
\hline
\end{tabular}




\subsubsection{Gas phase ROI}

The GROI includes the region from $\theta=20^{\circ}$ to $180^{\circ}$. If the full region is not present in the gas-phase FOV, the region from $\theta=20^{\circ}$ to the right-edge of the FOV is applied as the GROI. The GROI extends to a height $y_{\max }$ above the crest $\left(y_{\max }\right.$ is the crest height relative to the mean water level), i.e. $2 * y_{\max }$ above the mean water level.

The purpose of the GROI is to monitor the velocity field for signs of airflow separation. When the shear layer separates from the interface, there is a significant increase in the vorticity away from the interface, and a sheltered region of low air velocity between the interface and the separated shear layer. Two individual measures are included in order to monitor these properties in the GROI:

- The characteristic critical height, $\overline{y_{c}}$ is calculated by the area within the GROI with $u<C_{p}$, divided by the horizontal extent of the GROI. Hence, $\overline{y_{c}}$ represents the mean critical height (height where $u=C_{p}$ ) within the GROI. To remove the effect of wave amplitude we evaluate $\overline{y_{c}} / y_{\max }$ as a normalized measure of the sheltering.

- To evaluate the increased vorticity, we extract the rms vorticity within the GROI, denoted $\omega_{r m s, g}$. High values of $\omega_{r m s, g}$ are assessed to be related to the detachment of the shear layer and increased turbulence away from the gas-liquid interface.

When evaluating $\overline{y_{c}} / y_{\max }$ the GROI extends from the interface to its top border (region illustrated by dashed lines in figure 4$)$. When monitoring $\omega_{r m s, g}$, we omit the region closest to the interface (from the interface and $3 \mathrm{~mm} \mathrm{up}$ ), as we are interested in detecting signs of the high intensity shear layer separating from the interface, not shear which remains attached to the interface. Hence, the GROI for evaluating $\omega_{r m s, g}$ is bounded from below by the dotted line in figure 4 .

\section{Results}

\subsection{Example instantaneous velocity profiles}

Figure 5 presents instantaneous velocity fields for four waves (referred to as wave $A-D$ ). Figure 5 a) shows an example velocity field at $U_{s g}=1.85 \mathrm{~m} / \mathrm{s}$, while figure $5 \mathrm{~b}-\mathrm{d}$ ) are taken from the $U_{s g}$ $=2.20 \mathrm{~m} / \mathrm{s}$ experimental case. The figures present the velocity vectors in the air-phase, along with vorticity contours in both the gas and liquid phase, overlaid the original gas-phase PIV image. Note that the liquid vorticity is multiplied by 10 for visibility.

For all waves the negative shear layer is seen to remain attached above the wave crest. At the leeward side of the crest the shear layer is seen to separate from the interface, depending on the gas flow rate and wave crest geometry. This effect is most pronounced for the two steepest waves (figure $5 \mathrm{c}$ and d), but also for figure $5 \mathrm{~b}$ ) a slight detachment of the shear layer, and a sheltered region of low-velocity air is observed on the leeward side of the crest. In figure 5 c) and d) regions of negative axial velocity is observed within the sheltered region.

The vorticity contours from the liquid-phase PIV is also included in figure 5 (superimposed from the liquid phase PIV results). In figure $5 \mathrm{~b}$ ) and c) the vorticity is seen to be low, and evenly distributed along the wave profile, while in figure 5 a) and d) there are coherent regions of negative vorticity on the leeward side of the wave and below the crest. This indicates a turbulent spilling region caused by microscale wave breaking, and the waves are assessed to be in a state of active breaking according to the criterion presented in section 3.2. It can be noted that while the wave profile of figure $5 \mathrm{~b}$ ) is fairly smooth, parasitic capillaries are observed on the leeward side of the wave in figure $5 \mathrm{c}$ ). These capillary waves were also observed by Vollestad et al. (2019b) for steep non-breaking waves in the same experimental conditions. When a wave transitions into an active state of breaking, fluid elements on the leeward side of the crest spills downwards and the capillary waves are displaced downstream of the spilling region (Vollestad et al., 2019b). 


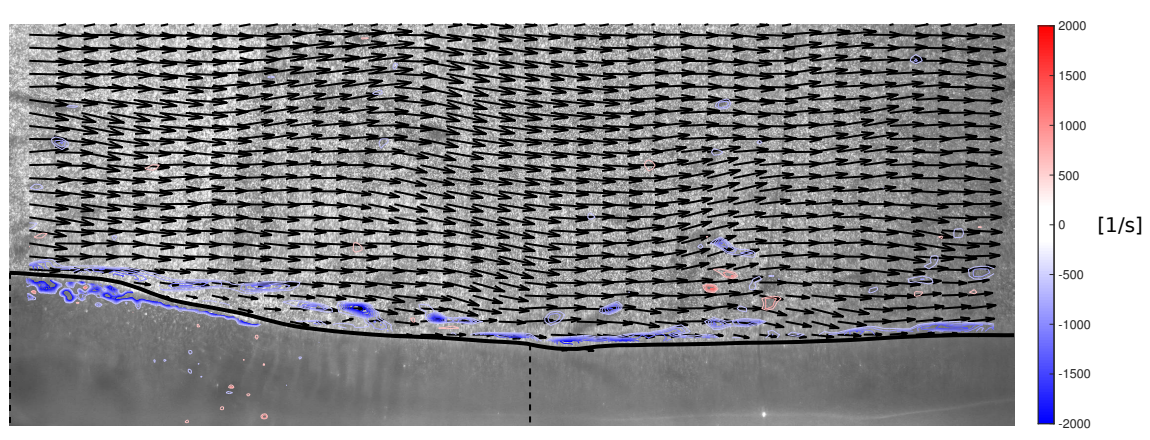

a) Wave $A, U_{s g}=1.85 \mathrm{~m} / \mathrm{s}$

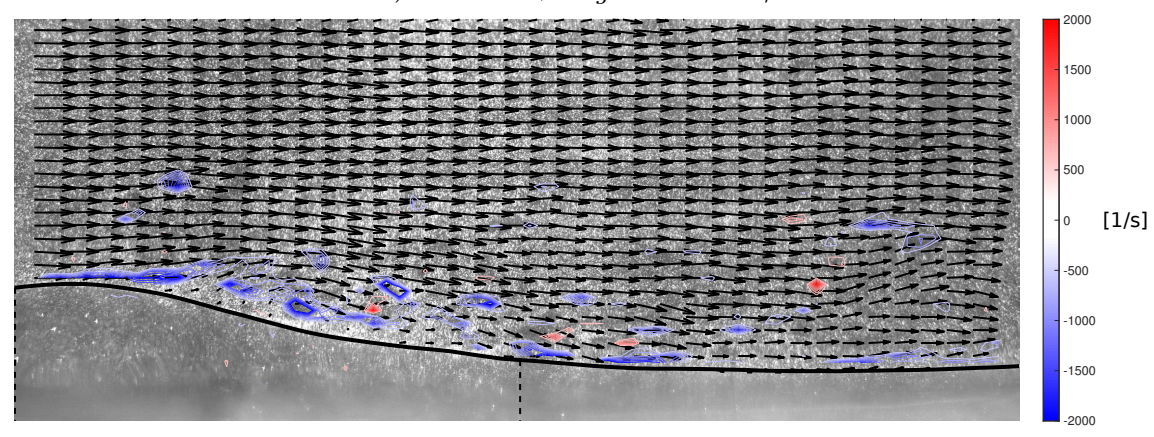

b) Wave $B, U_{s g}=2.20 \mathrm{~m} / \mathrm{s}$

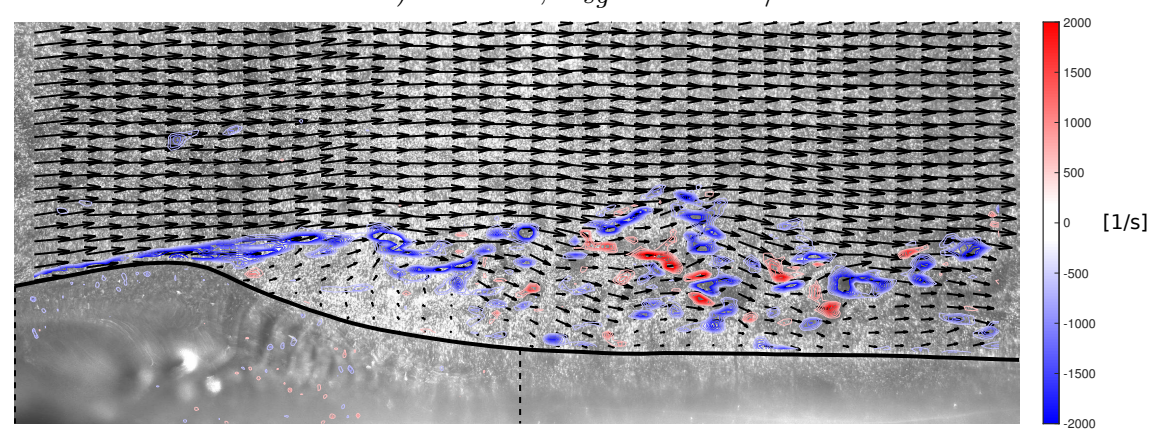

c) Wave $C, U_{s g}=2.20 \mathrm{~m} / \mathrm{s}$

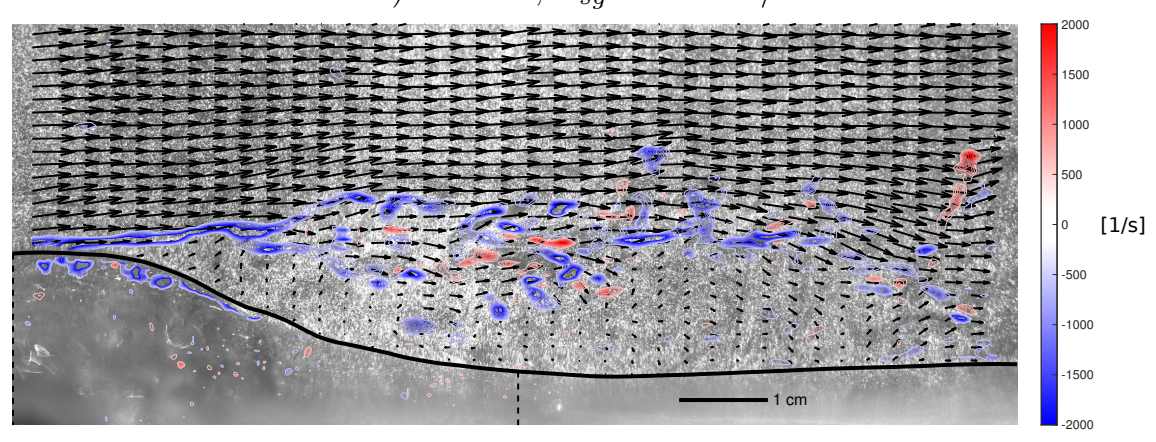

d) Wave $D, U_{s g}=2.20 \mathrm{~m} / \mathrm{s}$

Figure 5: Example velocity fields. Contours represent vorticity $\left[s^{-1}\right]$. NB: Liquid vorticity multiplied by 10 for visibility. Showing $1 / 4$ vectors in the gas phase. Dashed vertical lines indicate borders of liquid phase PIV. Solid black line: Interface. Length scale included in figure d. 
The interfaces of the four waves shown in figure 5 are presented in figure 6 . These were obtained from the LFV camera, and hence extend outside of the PIV FOV. Table 3 summarizes key parameters for the waves. Here we use the same notation as Reul et al. (2008). $y_{\max }$ and $y_{\min }$ is the maximum and minimum surface elevation, while $\lambda$ is the wavelength. $\epsilon_{\text {crest }}$ is the crest front-face (leeward side) steepness $\left(|\Delta y / \Delta x|\right.$ from the crest to the zero down-crossing at $\theta=90^{\circ}$ ), while $\delta_{\text {crest }}$ is the crest windward side steepness $\left(|\Delta y / \Delta x|\right.$ from $\theta=-90^{\circ}$ to $\left.0^{\circ}\right) . \mu$ is the vertical asymmetry factor $\left(\mu=y_{\max } /\left(y_{\max }+\left|y_{\min }\right|\right)\right)$, while $\lambda_{v}$ is the crest horizontal asymmetry factor $\left(\lambda_{v}=\epsilon_{\text {crest }} / \delta_{\text {crest }}\right)$. The wave steepness $a k$ is evaluated as $a k=\pi H / \lambda$, where $H$ is the wave height $\left(H=y_{\max }+\left|y_{\min }\right|\right)$.

From the data presented in table 3 it can be observed that there is only a moderate difference in the overall wave steepness $a k$ comparing the three waves at $U_{s g}=2.20 \mathrm{~m} / \mathrm{s}$. The front face steepness $\epsilon_{\text {crest }}$ is however significantly higher for wave $C$ and $D$.

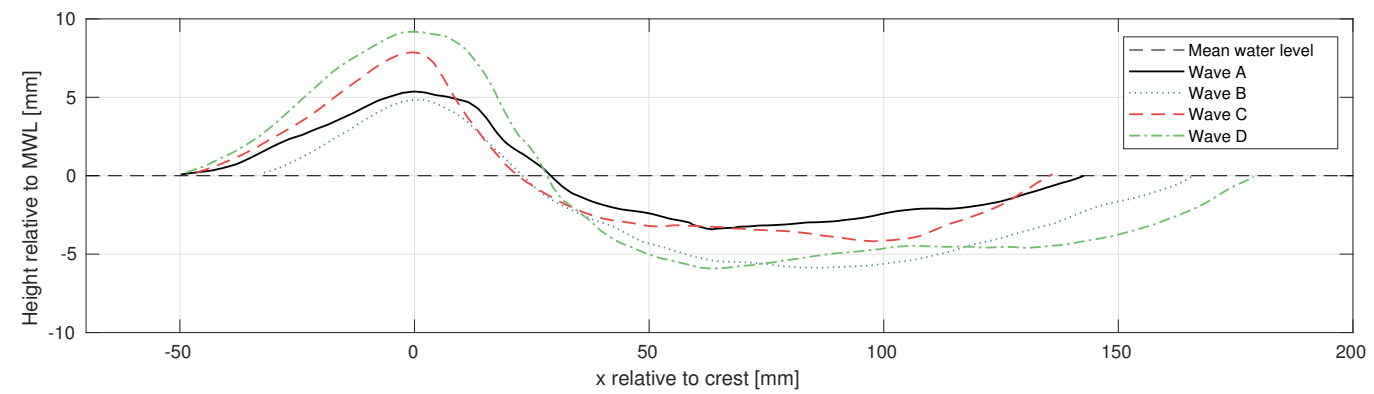

Figure 6: Interface elevation of the four waves displayed in figure 5 . Crest positioned at $\mathrm{x}=0$.

Table 3: Overview of parameters describing the wave interface for wave $A, B, C$ and $D$ presented in figure 6 .

\begin{tabular}{|c|c|c|c|c|c|c|c|c|c|}
\hline Wave & $y_{\max }[\mathrm{mm}]$ & $y_{\min }[\mathrm{mm}]$ & $\lambda[\mathrm{mm}]$ & $\epsilon_{\text {crest }}$ & $\delta_{\text {crest }}$ & $\mu$ & $\lambda_{v}$ & ak & Microbreaking \\
\hline$A$ & 5.3 & -3.4 & 193.0 & 0.19 & 0.11 & 0.61 & 1.73 & 0.15 & Yes \\
\hline$B$ & 4.9 & -5.9 & 198.0 & 0.21 & 0.15 & 0.45 & 1.40 & 0.17 & No \\
\hline$C$ & 7.9 & -4.2 & 182.5 & 0.35 & 0.17 & 0.65 & 2.06 & 0.21 & No \\
\hline$D$ & 9.2 & -5.9 & 227.5 & 0.32 & 0.20 & 0.61 & 1.60 & 0.21 & Yes \\
\hline
\end{tabular}

Table 4 presents the results for the parameters discussed in section 3.2. It can be seen that $\omega_{r m s, l}$ varies by a factor four from the non-breaking to breaking cases, and that following the criterion presented in section 3.2, wave $A$ and $D$ are in a state of active breaking, while $B$ and $C$ are non-breaking.

The normalized critical height is seen to increase through wave $A$ - $D$, from close to 0 for wave $A$ to 0.8 for wave $D$. This is in accordance with the visual impression obtained from figure 5 , as the sheltered region below the separated shear layer is seen to increase from wave $A$ to $D$. We observe that $\omega_{r m s, g}$ for wave $A$ is significantly lower than the three other waves. This is to be expected, as wave $A$ is from the $U_{s g}=1.85 \mathrm{~m} / \mathrm{s}$ case, where the shear on the gas-liquid interface is lower, and as the shear layer is seen to remain attached along the wave profile. While $\overline{y_{c}} / y_{\max }$ is maximum for wave $D, \omega_{r m s, g}$ is higher for wave $C$. Comparing figure 5 c) and d) the region of high intensity shear is seen to be somewhat thicker above wave $C$, indicating a higher degree of turbulent mixing.

The results in this section provide some indications of the importance of the crest geometry to the structure of the airflow. However, analyzing the effect of individual wave profile parameters and the effect of breaking requires a statistical approach. 
Table 4: Overview of results from the ROI analysis for the four waves.

\begin{tabular}{|c|c|c|c|c|}
\hline Wave & $\omega_{r m s, l}\left[s^{-1}\right]$ & $\omega_{r m s, g}\left[s^{-1}\right]$ & $\overline{y_{c}}[\mathrm{~mm}]$ & $\overline{y_{c}} / y_{\max }[-]$ \\
\hline$A$ & 168.6 & 268 & 0.1 & 0.02 \\
\hline$B$ & 43.9 & 560 & 1.0 & 0.21 \\
\hline$C$ & 44.6 & 739 & 4.1 & 0.52 \\
\hline$D$ & 176.4 & 689 & 7.4 & 0.80 \\
\hline
\end{tabular}

\subsection{Airflow statistics over breaking and non-breaking waves}

In this section, statistics of $\overline{y_{c}} / y_{\max }$ and $\omega_{r m s, g}$ above breaking and non-breaking waves are presented. To isolate the effect of active wave breaking from the geometrical properties of the waves, it is necessary to keep at least one of the geometrical properties of the waves constant, as wave geometry obviously has a significant impact on the airflow.

Reul et al. (2008) characterized the extent of the separated region by the air velocity, crest height and $\epsilon_{\text {crest }}$. Here, we isolate the air velocity by comparing each of the experimental cases individually. As the vertical extent of the GROI used to evaluate $\omega_{r m s, g}$, and $\overline{y_{c}} / y_{\max }$ is scaled by the local crest height, $\epsilon_{\text {crest }}$ is chosen as the geometrical property of the wave to keep constant. Note that the average wave profile of breaking and non-breaking waves with the same $\epsilon_{\text {crest }}$ will vary, as wave breaking modifies the geometrical properties of the waves. Hence, completely isolating the effect of active wave breaking from the geometrical properties of individual waves is not possible. The variations on the overall wave form for breaking and non-breaking waves at constant $\epsilon_{\text {crest }}$, and its effect on the results is discussed in section 5 .

Breaking and non-breaking waves were evaluated individually, and binned in $\epsilon_{\text {crest }}$ intervals of 0.05. For each $\epsilon_{\text {crest }}$-interval with more than five observations the mean value, standard deviation and $95 \%$ confidence interval of the mean value (evaluated by the t-distribution) of $\overline{y_{c}} / y_{\max }$ and $\omega_{r m s, g}$ is evaluated. Results are presented in figure 7 .

The results in figure 7 show that both $\omega_{r m s, g}$ and $\overline{y_{c}} / y_{\max }$ is strongly correlated with $\epsilon_{\text {crest }}$. As expected, increased $\epsilon_{\text {crest }}$ is seen to result in increased turbulence and larger areas of sheltered airflow on the leeward side of the crest. It can be noted that all waves with $\epsilon_{\text {crest }}<0.1$ are found to be non-breaking, while the steepest waves at $U_{s g}=2.20 \mathrm{~m} / \mathrm{s}$ are found to be breaking. The shaded area represents the standard deviation around the mean value, and significant deviations around the mean value are observed both for breaking and non-breaking waves.

Results for the $U_{s g}=2.20 \mathrm{~m} / \mathrm{s}$ case shows that the breaking and non-breaking cases are more or less overlapping. The $95 \%$ confidence intervals are everywhere overlapping, indicating that while there are some indications that $\omega_{r m s, g}$ is higher for breaking waves within the steepness interval of $0.1<\epsilon_{\text {crest }}<0.25$, these results are not considered statistically significant.

At $U_{s g}=1.85 \mathrm{~m} / \mathrm{s}$ there is a significant difference in the statistics above breaking and nonbreaking waves. In the interval $0.2<\epsilon_{\text {crest }}<0.35$, the non-breaking waves are observed to results in significantly higher $\omega_{r m s, g}$ and $\overline{y_{c}} / y_{\max }$. This indicates that within this steepness interval, active wave breaking reduces the intensity of shear layer separation, as both the sheltered region and the turbulence generated by the separated shear layer is reduced. This is a somewhat surprising result as wave breaking is typically assessed to enhance the airflow separation above waves.

While the results presented in this section are indicative of the effect of active wave breaking for the cases considered, further analysis of the experimental data is needed to understand how the variations in $\omega_{r m s, g}$ and $\overline{y_{c}} / y_{\max }$ observed for the $U_{s g}=1.85 \mathrm{~m} / \mathrm{s}$ case manifests itself in the average flow field above breaking and non-breaking waves. 


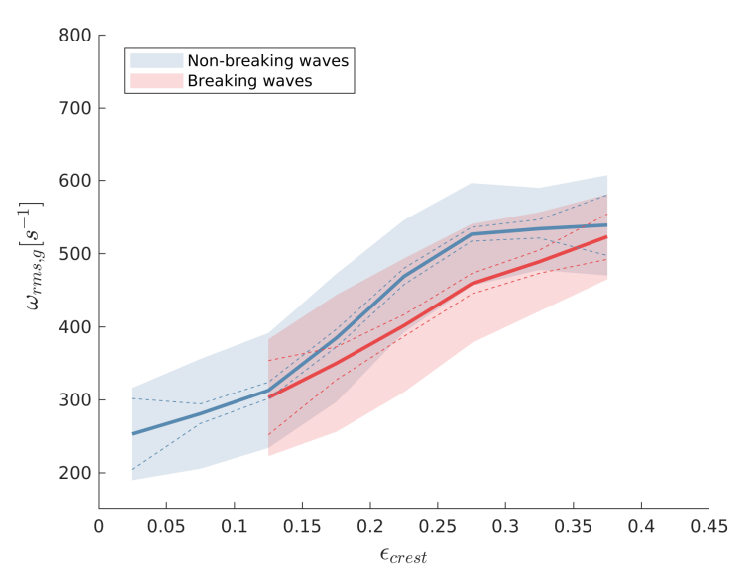

a) $U_{s g}=1.85 \mathrm{~m} / \mathrm{s}$

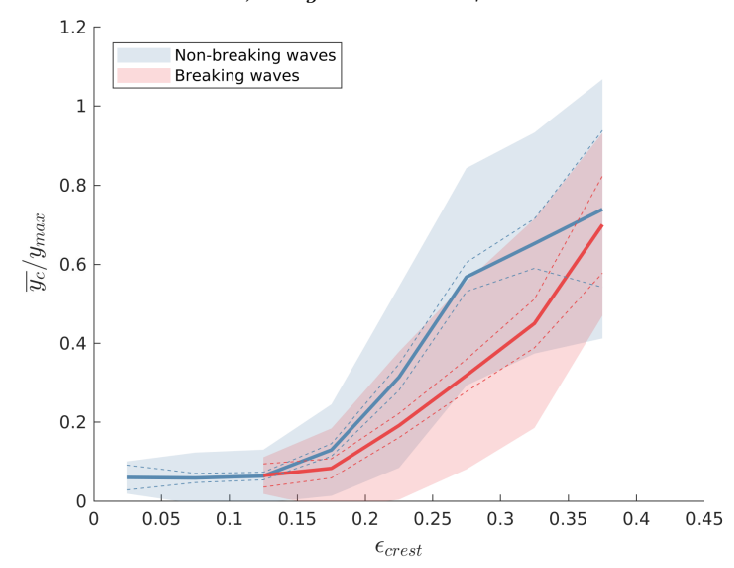

c) $U_{s g}=1.85 \mathrm{~m} / \mathrm{s}$

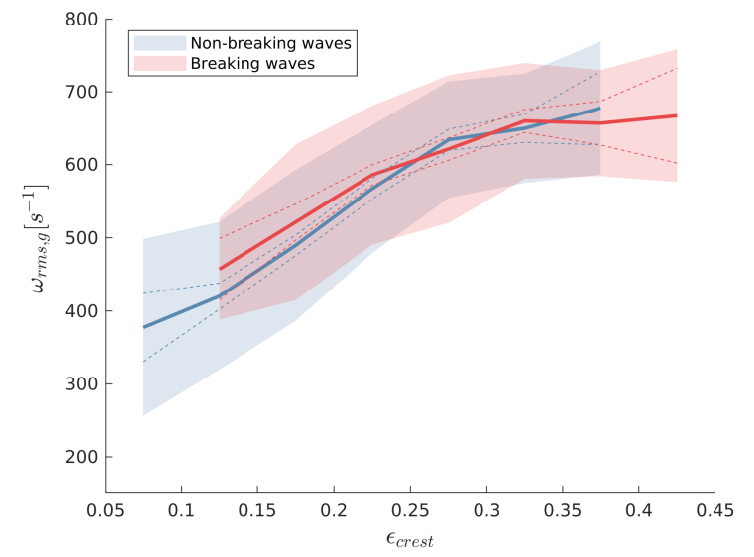

b) $U_{s g}=2.20 \mathrm{~m} / \mathrm{s}$

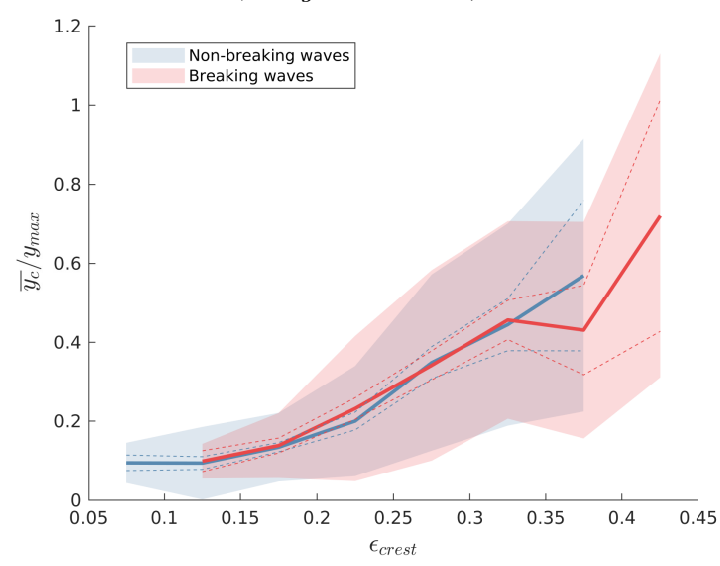

d) $U_{s g}=2.20 \mathrm{~m} / \mathrm{s}$

Figure 7: Statistics of $\omega_{r m s, g}(\mathrm{a}, \mathrm{b})$ and $\overline{y_{c}} / y_{\max }(\mathrm{c}, \mathrm{d})$ as a function of $\epsilon_{\text {crest }}$. Thick full line: mean value, dashed line: $95 \%$ confidence interval of mean value, shaded color: mean value \pm standard deviation. $U_{s g}=1.85 \mathrm{~m} / \mathrm{s}(\mathrm{a}, \mathrm{c})$, and $U_{s g}=2.20 \mathrm{~m} / \mathrm{s}(\mathrm{b}, \mathrm{d})$.

\subsection{Analysis of effect of breaking for $0.225<\epsilon_{\text {crest }}<0.325$}

In this section we perform an analysis of the velocity fields above breaking and non-breaking waves within the steepness interval $0.225<\epsilon_{\text {crest }}<0.325$, i.e. within the interval where we in section 4.2 observed a reduced sheltering due to active wave breaking in the $U_{s g}=1.85 \mathrm{~m} / \mathrm{s}$ case. The analysis is performed both for the $U_{s g}=1.85$ and $2.20 \mathrm{~m} / \mathrm{s}$ case, to further describe the effect of active wave breaking observed in section 4.2. In section 4.3.1 a conditional phase-averaging of the velocity fields is performed, while in section 4.3.2 the spectra above breaking and non-breaking waves are analyzed.

While $\epsilon_{\text {crest }}$ is kept constant, other properties of the waves will vary slightly comparing breaking and non-breaking waves. The mean properties of the waves under investigation are presented in table 5. It is observed that while the average front-face steepness $\epsilon_{\text {crest }}$ is equal, the average crest height $y_{\max }$ and wave height $H$ for non-breaking waves is somewhat higher than for the breaking waves. These variations will be discussed in section 5 .

\subsubsection{Phase-averaged results}

A conditional phase-averaging of the gas and liquid phase is performed. The wave-following coordinate system is described in Appendix A. The coordinate system in the liquid phase is the same as the coordinate system employed by Vollestad et al. (2019b) to evaluate phase-averaged statistics of the liquid phase of microscale breaking waves, while the wave-following coordinate system in the 
Table 5: Mean properties of the four averaging cases under investigation.

\begin{tabular}{|c|c|c|c|c|c|c|}
\hline$U_{s g}$ & State & $\overline{\epsilon_{\text {crest }}}$ & $\overline{y_{\max }}$ & $\bar{H}[\mathrm{~mm}]$ & $\bar{\lambda}[\mathrm{mm}]$ & $\overline{a k}$ \\
\hline 1.85 & non-breaking & 0.27 & 6.5 & 11.1 & 181 & 0.19 \\
\hline 1.85 & breaking & 0.27 & 6.1 & 10.5 & 181 & 0.18 \\
\hline 2.20 & non-breaking & 0.27 & 7.5 & 13.0 & 258 & 0.16 \\
\hline 2.20 & breaking & 0.27 & 7.1 & 12.1 & 222 & 0.17 \\
\hline
\end{tabular}

gas phase is similar to the coordinate system used by Vollestad et al. (2019a), analyzing airflow over waves.

Figure 8 and 9 presents the phase-averaged horizontal velocity $(\langle u\rangle)$ for the $U_{s g}=1.85$ and $2.20 \mathrm{~m} / \mathrm{s}$ case respectively. Breaking and non-breaking waves are averaged individually. Due to the limited FOV of the PIV cameras and the triggering applied (ref. section 2), the region where we can obtain reliable phase-averaged velocity profiles is limited to approximately $-10^{\circ}<\theta<180^{\circ}$ for the gas phase and $-10^{\circ}<\theta<120^{\circ}$ for the liquid phase. Here more than 100 datapoints are used in the evaluation of each phase-averaged quantity. In the range $20^{\circ}<\theta<80^{\circ}$ (LROI), more than 200 datapoints are obtained (number decreasing with distance from LROI). While the phase-averaged velocity profiles are not fully converged, the main flow features, such as the extent of the sheltered region, are assessed to be qualitatively converged, as performing the averaging with half of the available data resulted in only minor deviations from the results presented in figure 8 and 9 , and no qualitative change in the results or conclusions related to the phase-averaged flow fields.

The results are overlaid the average interface (evaluated individually for breaking and nonbreaking waves) detected by the LFV camera. Isocurves of constant velocity are included in the gas-phase velocity field. The critical height (where $\langle u\rangle=C_{p}$ ) is illustrated by a red dashed line.

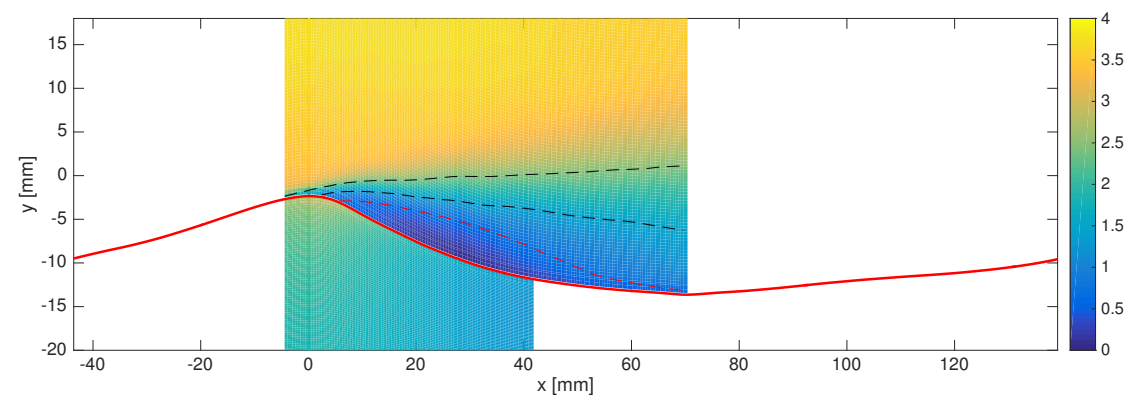

a) $U_{s g}=1.85 \mathrm{~m} / \mathrm{s}$, non-breaking

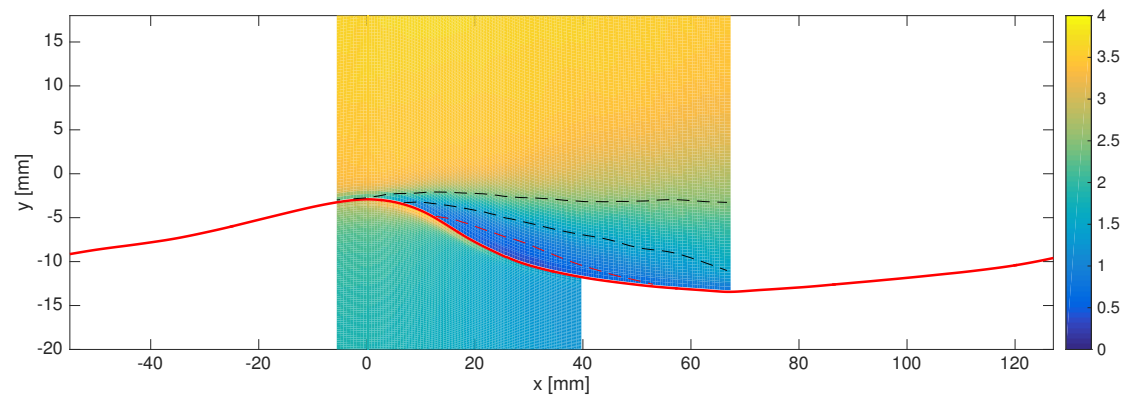

b) $U_{s g}=1.85 \mathrm{~m} / \mathrm{s}$, breaking

Figure 8: Phase-averaged results for the horizontal velocity $\langle u\rangle$, converted to $(x, y)$-coordinates by applying the mean interface elevation for each case. Results plotted for $-10^{\circ}<\theta<180^{\circ}$. Liquid phase horizontal velocity multiplied by 5 for visibility. Colorbar in $[\mathrm{m} / \mathrm{s}]$. Full red line indicate mean interface elevation. Red dashed line represent the critical height, where $\langle u\rangle=C_{p}$. Black dashed lines represent isocurves at 1.5 and $2.5 \mathrm{~m} / \mathrm{s}$. 


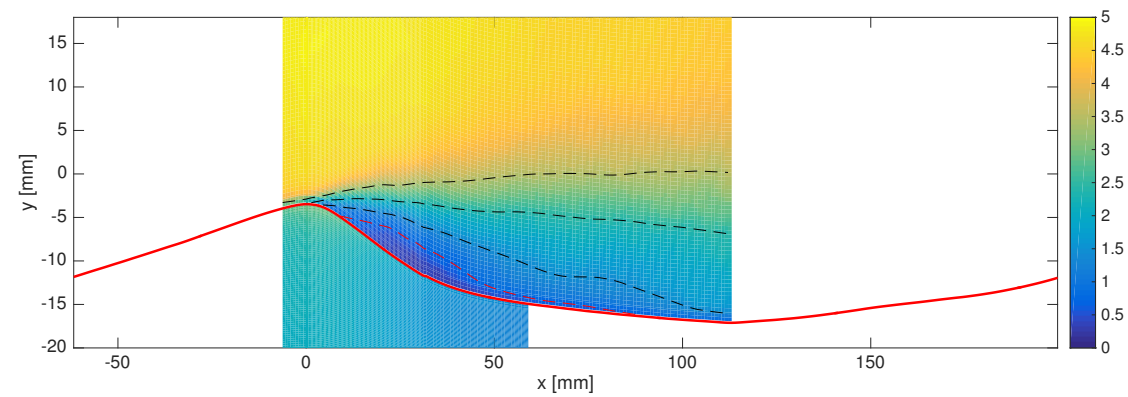

a) $U_{s g}=2.20 \mathrm{~m} / \mathrm{s}$, non-breaking

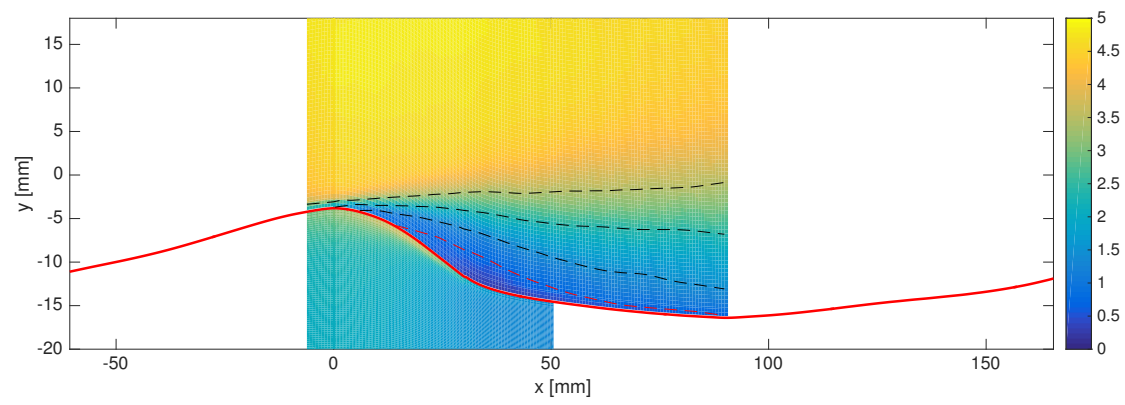

b) $U_{s g}=2.20 \mathrm{~m} / \mathrm{s}$, breaking

Figure 9: Phase-averaged results for the horizontal velocity $\langle u\rangle$, converted to $(x, y)$-coordinates by applying the mean interface elevation for each case. Results plotted for $-10^{\circ}<\theta<180^{\circ}$. Liquid phase horizontal velocity multiplied by 5 for visibility. Colorbar in $[\mathrm{m} / \mathrm{s}]$. Full red line indicate mean interface elevation. Red dashed line represent the critical height, where $\langle u\rangle=C_{p}$. Black dashed lines represent isocurves at $1.5,2.5$ and $3.5 \mathrm{~m} / \mathrm{s}$.

The results in figure 8 show that the airflow is more sheltered in the lee of the non-breaking waves compared with the breaking waves. While the critical height is seen to detach from the interface close to the crest for the non-breaking case, the detachment occurs later over breaking waves, and the area where $\langle u\rangle\left\langle C_{p}\right.$ is significantly reduced. It can be noted that while the peak horizontal velocity in the liquid phase of the non-breaking wave is centered below the crest, the peak is shifted towards the leeward side of the breaking waves. This is a clear indication of the spilling region observed below breaking waves. One interpretation of the results is that the spilling motion of the liquid on the leeward side of the wave reduces the shear on the gas flow, or even helps accelerate the gas close to the surface as the spilling is initiated, thus reducing the sheltered region on the leeward side of the wave.

The results presented in figure 9 shows that the effect of breaking is less pronounced for the $U_{s g}=2.20 \mathrm{~m} / \mathrm{s}$ case compared with the lower gas flow rate case. Again the critical height is seen to detach from the interface somewhat further downwind on the wave profile for the breaking case, but the sheltered area (here evaluated as the area limited by $\langle u\rangle\left\langle C_{p}\right.$ ) is very similar, comparing the breaking and non-breaking case.

In figure 10, streamlines of the phase-averaged velocity fields, observed in a frame of reference moving with the characteristic wave speed $C_{p}$, is presented for the $U_{s g}=1.85 \mathrm{~m} / \mathrm{s}$ case. For both the breaking and non-breaking wave case a clear "cat's eye" structure is observed on the leeward side of the crest. This illustrates that as the waves propagate in the streamwise direction, they experience a co-moving pocket of air in its front, displacing the streamlines in the outer flow relative to the wave profile. In table 6 the location of the cat's eye center $\left(\theta_{c}, \xi_{c}\right.$, where $\xi_{c}$ is the height above the interface) and the area under the critical height $y_{c}$ is summarized for all four averaging cases. The results show that while the sheltered area below non-breaking waves is slightly larger compared with the breaking waves at $U_{s g}=2.20 \mathrm{~m} / \mathrm{s}$, the effect is much more pronounced for the lower gas flow 
rate, and the area below the critical height is observed to be three times larger for the non-breaking waves at $U_{s g}=1.85$ compared with breaking waves of similar $\epsilon_{\text {crest }}$. These results are consistent with the results obtained in section 4.2 , where it was found that $\overline{y_{c}} / y_{\max }$ was significantly reduced as a result of breaking for the $U_{s g}=1.85 \mathrm{~m} / \mathrm{s}$ case.

Gent \& Taylor (1977) included an increased surface drift velocity to numerically analyze the effect of wave breaking on the extent of the cat's eye center. Analyzing a strongly forced wave $\left(c / u_{*} \approx 1.5\right)$ they found that the effect of the increased surface drift was to reduce the elevation of the cat's eye center by approximately $30 \%$. While this is qualitatively in agreement with the results observed for the $U_{s g}=1.85 \mathrm{~m} / \mathrm{s}$ case, the waves considered by Gent \& Taylor (1977) has an even lower wave age than the cases considered in this work, hence the $U_{s g}=2.20 \mathrm{~m} / \mathrm{s}$ case should be the more comparable. However, here we do not observe any significant change in the elevation of the cat's eye center.

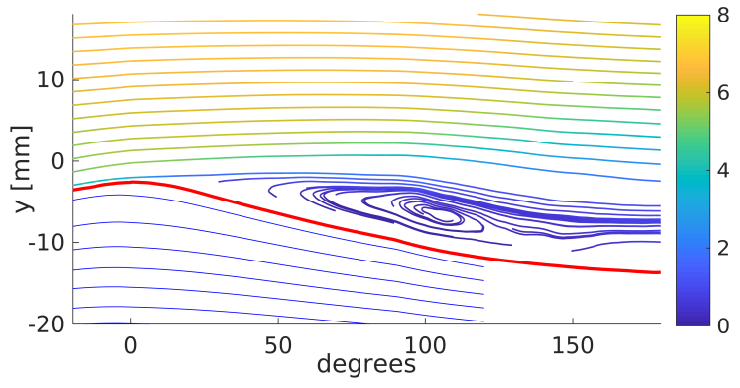

a) Non-breaking

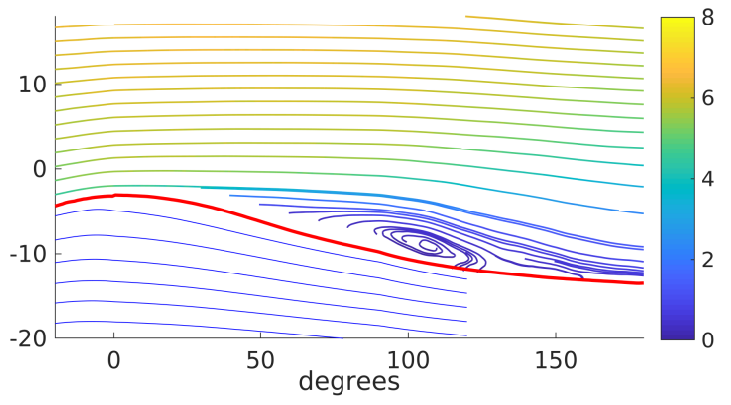

b) Breaking

Figure 10: Streamlines of phase-averaged velocity fields seen in a frame of reference moving with the characteristic wave speed $C_{p}\left(\langle\mathbf{u}\rangle-C_{p}\right)$ for the $U_{s g}=1.85 \mathrm{~m} / \mathrm{s}$ case. Gas-phase streamlines colored by $0.5\left(\langle u\rangle^{2}+\langle v\rangle^{2}\right)\left[m^{2} / s^{2}\right]$.

Table 6: Phase-averaged results. $\theta_{c}$ and $\xi_{c}$ is the location (wave phase and height above the interface) of the cat's eye center.

\begin{tabular}{|c|c|c|c|c|}
\hline$U_{s g}[\mathrm{~m} / \mathrm{s}]$ & State & $\theta_{c}$ (degrees) & $\xi_{c}[\mathrm{~mm}]$ & Area where $\langle u\rangle<C_{p}\left[\mathrm{~mm}^{2}\right]$ \\
\hline 1.85 & non-breaking & 107 & 4.4 & 150 \\
\hline 1.85 & breaking & 108 & 2.1 & 49 \\
\hline 2.20 & non-breaking & 83 & 3.5 & 113 \\
\hline 2.20 & breaking & 96 & 3.5 & 103 \\
\hline
\end{tabular}

The tangential velocity $u_{t}$ along the interface was evaluated for each PIV velocity field, and phase-averaged similarly to the horizontal and vertical velocity components. Due to limitations in the spatial resolution and the accuracy of the interface detection, we are not able to evaluate the tangential shear in the viscous sublayer. Instead we evaluate the average tangential velocity over the two velocity vectors closest to the interface, in both the gas and liquid phase, and compute $\Delta u_{t} / \Delta y$ across the interface. While this does not provide the shear stresses directly, the results are indicative of the development of the shear stress along the wave profiles. Results are presented in figure 11. Note that the results are plotted for $-10^{\circ}<\theta<120^{\circ}$, as reliable phase-averaged velocities in both the gas and liquid phase are required.

The results presented in figure 11 indicate that the shear stress at the interface varies considerably between the four averaging cases. $\Delta u_{t} / \Delta y$ drops off faster for the non-breaking compared to the breaking waves, and for the non-breaking waves at $U_{s g}=1.85 \mathrm{~m} / \mathrm{s}$ the results shows that there is a significant region of the wave profile $\left(50^{\circ}<\theta<110^{\circ}\right)$ where the liquid on average travels faster than the overlying air, and will exert a force on the gas phase in the streamwise direction.

From figure 11 we also can observe that the velocity gradient across the interface at the crest 


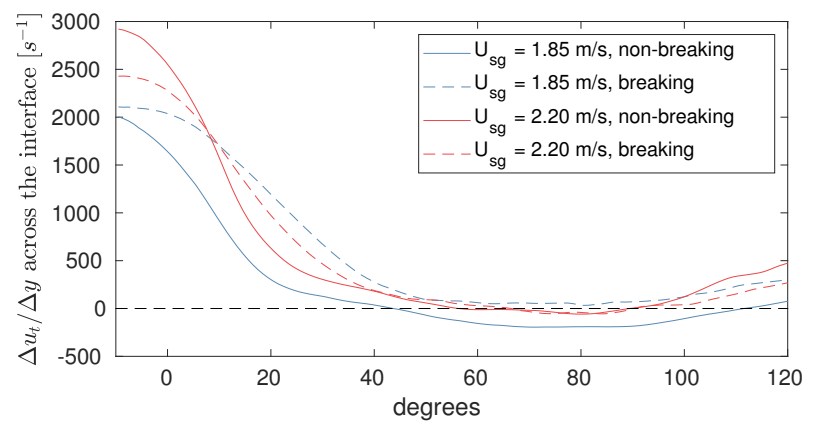

Figure 11: $\Delta u_{t} / \Delta y$ over the interface, plotted in the interval $-10^{\circ}<\theta<120^{\circ}$.

is higher for non-breaking than breaking waves at $U_{s g}=2.20 \mathrm{~m} / \mathrm{s}$. Analyzing the phase-averaged velocity fields, it was found that the main reason for this discrepancy was a higher gas velocity close to the interface for the non-breaking waves, not increased liquid velocity caused by wave breaking. The reason for this behavior is not completely understood, but it might be related to the somewhat higher crest-height of the non-breaking waves compared with the breaking waves under investigation (ref. table 5), as the lower amplitude breaking waves may to a larger degree be sheltered by upwind waves. It is also possible that local interface variations on the windward side of the wave is the reason for these deviations, as the phase-averaged interface profiles indicate that the non-breaking waves are locally steeper close to the wave crest of the windward side compared with the breaking waves. This may result in a higher acceleration of the flow near the crest for non-breaking compared with breaking waves at $U_{s g}=2.20 \mathrm{~m} / \mathrm{s}$.

The momentum flux at the interface is governed both by shear stresses and form drag. As the experimental setup does not facilitate measurements of the pressure along the interface, we are not able to infer the overall change in the momentum flux as a result of active breaking. As we consider young waves the critical height is located within the inner region, where the turbulent eddies are in local equilibrium (Belcher \& Hunt, 1993, 1998). In this region the turbulent shear stresses displace the streamlines asymmetrically, and the phase shift of the pressure field can not be inferred directly from the location of the cat's eye (Kihara et al., 2007).

From the air-sea literature it is well established that form drag contributes to the majority of the wind stress at the wave ages considered in this work (Banner \& Peirson, 1998; Sullivan et al., $2018 b, a)$. While the pipe walls will influence the pressure distribution relative to the pressure field above waves in an open system, it is considered likely that this will also be the case for the current experimental investigation. Hence the total momentum flux may well be higher above non-breaking compared with breaking waves at $U_{s l}=1.85 \mathrm{~m} / \mathrm{s}$, despite the reduced shear stress.

\subsubsection{Spectra above breaking and non-breaking waves}

The results in section 4.2 showed that the $\omega_{r m s, g}$ was higher over non-breaking than breaking waves at $U_{s g}=1.85 \mathrm{~m} / \mathrm{s}$. This is assessed to be related to increased turbulence intensity on the leeward side of the wave. To further investigate the turbulence above breaking and non-breaking waves, the wavenumber spectra of the horizontal velocity fluctuations are evaluated above breaking and non-breaking waves individually. Again we limit the cases under investigation to a steepness interval of $0.225<\epsilon_{\text {crest }}<0.325$. The horizontal velocity $1 \mathrm{~mm}$ above the wave crest is extracted for each wave. The periodogram is evaluated for each velocity profile and the mean of the periodogram is used to estimate the horizontal velocity spectra. Results are presented in figure 12 .

From the spectra it can be observed that at $U_{s g}=1.85 \mathrm{~m} / \mathrm{s}$, the airflow is more turbulent over non-breaking than breaking waves. At $U_{s g}=2.10 \mathrm{~m} / \mathrm{s}$ the spectra over breaking and non-breaking waves are seen to be more or less equal. This was also observed when the spectra were evaluated 


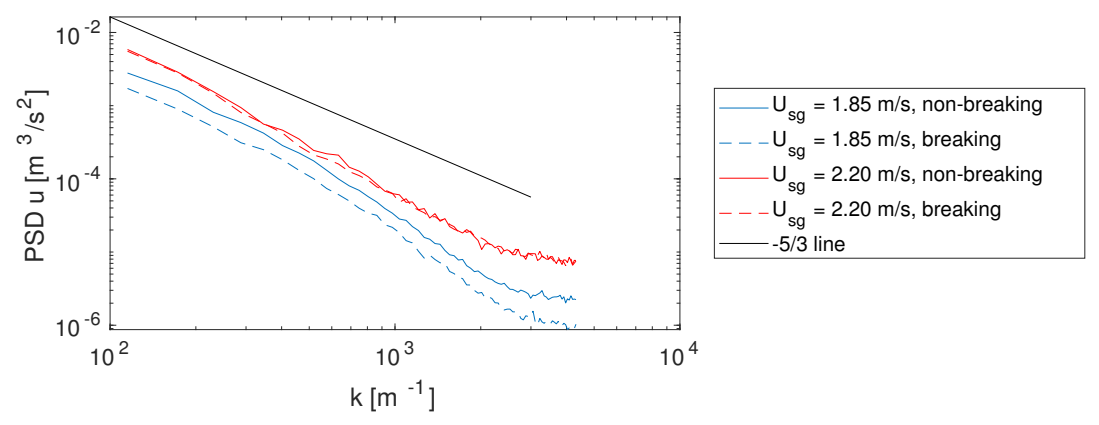

Figure 12: Power spectral density of the horizontal velocity component (PSD u), evaluated $1 \mathrm{~mm}$ above the crest of each wave observed with $0.225<\epsilon_{\text {crest }}<0.325$.

at a fixed height $(\mathrm{y}=5 \mathrm{~mm})$.

These results are consistent with the results obtained in section 4.2 , and indicate that active wave breaking has the effect of reducing the turbulence levels above the wave trough for moderate wind speeds. We relate this to a reduction in the frequency and/or extent of airflow separation on the leeward side of the crest, which could be a result of a change in the wave geometry as the waves transition from non-breaking to breaking, and/or of the spilling motion induced by wave breaking (this will be discussed in section 5). At the higher airflow rate considered $\left(U_{s g}=2.20 \mathrm{~m} / \mathrm{s}\right.$ ), the effect is not observed, and again we conclude that active wave breaking does not significantly influence the airflow above waves at $U_{s g}=2.20 \mathrm{~m} / \mathrm{s}$.

\section{Discussion}

In this study efforts have been made to isolate the effect of wave breaking, by comparing breaking and non-breaking waves with similar geometrical properties (with focus on $\epsilon_{\text {crest }}$ ). However, completely isolating the effect of the turbulent spilling region of the liquid is not possible, as wave breaking modifies the interface of the waves. In figure 13 the phase-averaged interface for the four averaging cases analyzed in section 4.3 is presented. Results are presented for $\theta$ in the range of $-20^{\circ}$ to $180^{\circ}$.

Figure 13 shows that while the waves are evaluated within a narrow $\epsilon_{\text {crest }}$-band, there is a distinct difference in the average interface shape of breaking and non-breaking waves. The non-breaking waves are observed to be somewhat taller, with a higher local steepness close to the wave crest compared with the breaking waves at similar $\epsilon_{\text {crest }}$. The more rounded profile of the breaking waves may help explain why we observe a reduced sheltered region on the leeward side of the breaking waves at $U_{s g}=1.85 \mathrm{~m} / \mathrm{s}$, as the more gentle slope of the interface may facilitate flow expansion without separation close to the crest. A more direct explanation for the observations made is that the spilling motion of the liquid (observed in the phase-averaged velocity profiles presented in figure 8 and 9) reduces the interfacial shear on the leeward side of the wave, preventing flow separation. Isolating these effects (the spilling motion induced by breaking and the change in wave shape also due to breaking) is not possible when considering naturally occurring wind-generated waves. Numerical analysis or controlled experiments with artificially generated waves is necessary to evaluate which of these effects is dominant in reducing the sheltered region behind breaking waves at the lower gas flow rate.

It is important to note that all the results in the present work are obtained from, and only valid for, the center plane of a two-phase pipe flow configuration. The closed pipe geometry induces lateral velocity variations which are not found in open wind-wave tanks, and the wave-coherent pressure variations at the upper pipe wall will be reflected down, modifying the wave coherent 


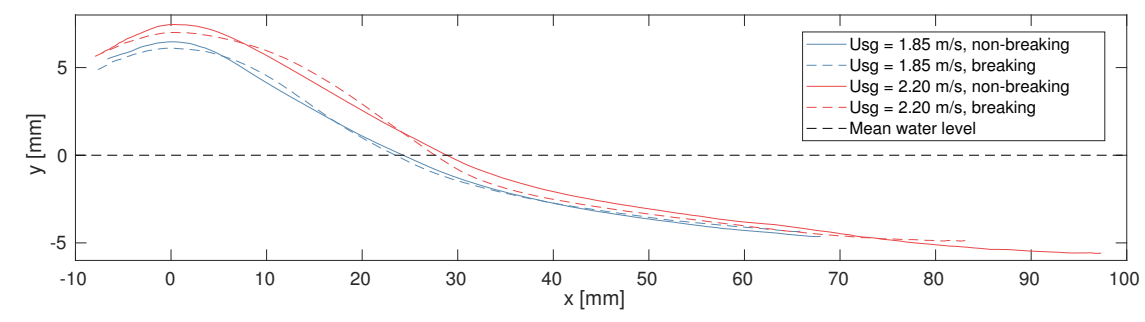

Figure 13: Mean interface of averaging cases, interval from $\theta=-20^{\circ}$ to $180^{\circ}$ converted to world coordinates.

pressure field relative to airflow over waves in an open channel. However, in the trough-to-crest region of the pipe centerline (where we focus our attention) the airflow is primarily influenced by the gas-liquid interface (as the distance to the pipe walls are much larger than the distance to the interface), and the lateral variations are much smaller than in the cross-sections near the pipe walls. As the relationship between active wave breaking and airflow separation has not previously been investigated in a pipe flow configuration, and as the wave breaking process and airflow above waves in pipes has previously been found to have many features similar to air-sea interactions (Vollestad et al., 2019a,b), it is considered expedient to compare (in a qualitative manner) the results obtained in this work with studies conducted in an open channel.

Numerical results by Sullivan et al. (2018b), simulating wind over waves representing incipient and active breaking, indicate that the overall wave steepness, not the increased drift velocity or the local steepness variations between incipient and actively breaking waves, is the main parameter controlling the form drag. The simulations by Sullivan et al. (2018b) are for a wave age which is even lower than the $U_{s g}=2.20 \mathrm{~m} / \mathrm{s}$ case, hence the effect of active wave breaking observed for the $U_{s g}=1.85 \mathrm{~m} / \mathrm{s}$ case is not necessarily in contradiction with the numerical results by Sullivan et al. (2018b). The results in this work indicate that the effect of wave breaking is relatively more important to the overlying airflow at lower gas flow rates.

Reul et al. (2008) attributed the change in the separated flow region to the front face steepness $\left(\epsilon_{\text {crest }}\right)$. It is interesting to note their figure 10 , depicting two waves at $c / u_{*} \approx 2.6$ (closely matching the $U_{s g} 1.85 \mathrm{~m} / \mathrm{s}$ case). While the airflow clearly separates over one wave at incipient breaking $\left(\epsilon_{\text {crest }}=0.52\right)$, it remains attached over the other wave profile $\left(\epsilon_{\text {crest }}=0.28\right)$, which appears to be in a state of active wave breaking. While Reul et al. (2008) observe a clear correlation between both the horizontal and vertical extent of the separation bubble with $\epsilon_{\text {crest }}$ (which one can expect also from the results presented in section 4.2 in the present work), there is considerable scatter in the results, and the results for the $U_{s g}=1.85 \mathrm{~m} / \mathrm{s}$ case in the present work could indicate that the state of breaking, as well as the crest front face steepness influences the extent of the separated zone. This was however not investigated by Reul et al. (2008).

Investigating the effect of wave breaking on even lower gas flow rates would be of interest. However, as the first signs of microscale wave breaking is observed at $U_{s g}=1.80 \mathrm{~m} / \mathrm{s}$ (Vollestad et al., 2019b), this would require a different experimental setup.

\section{Concluding remarks}

In this work we have performed simultaneous two-phase PIV with the aim to detect the influence of microbreaking on the airflow above waves in stratified gas-liquid pipe flow. Using the criterion for separating actively breaking and non-breaking waves by Vollestad et al. (2019b), we analyze the effect of active wave breaking from microscale breaking waves on the airflow structure.

Results show that at the lowest gas flow rate considered $\left(U_{s g}=1.85 \mathrm{~m} / \mathrm{s}\right)$, wave breaking has a stabilizing effect on the airflow above the waves, reducing the extent of the separated flow region and the turbulence above the waves. Hence, the effect of airflow separation is reduced over actively 
breaking waves at this moderate flow rate. At $U_{s g}=2.20 \mathrm{~m} / \mathrm{s}$ the effect of wave breaking is observed to be more moderate. The results indicate that the region in the flow map where active wave breaking significantly influences the air flow field in two-phase pipe flow is relatively small.

While a direct application of the results is restricted to a two-phase pipe flow configuration, the results can also be related to phenomena occurring in the ocean. The impact of wave breaking on the airflow is still a topic under investigation, and the present work adds new insight into the coupling between small scale wave breaking events and airflow separation at high wind forcing.

\section{Acknowledgement}

The authors wish to acknowledge the strategic research initiative EarthFlows at the Faculty of Mathematics and Natural Sciences at the University of Oslo. Laboratory Head Engineer Olav Gundersen is gratefully acknowledged for the technical assistance he provided for the experimental work.

\section{Appendix}

\section{Appendix A: Phase-averaging procedure}

The wave-following coordinate system applied is illustrated in figure 14 . The wave phase $\theta$ (evaluated by the zero-crossing procedure described in section 3.2) is used together with a wavefollowing $\zeta$-coordinate to obtain a wave-following coordinate system. The $\zeta$-coordinate is implemented slightly differently in the two phases. In the liquid phase, $\zeta_{l}$ is evenly distributed over 100 points from the lower part of the PIV velocity field (at $\mathrm{y}=-28 \mathrm{~mm}$ ) to the interface. This is similar to the wave-following coordinate system applied by Vollestad et al. (2019b). In the gas phase the wave amplitude is a critical parameter, impacting on the extent of the sheltered region. For this reason the $\zeta_{g}$-coordinate is normalized by the wave crest height. $\zeta_{g}$ is evenly distributed over 100 points, from the interface to a height $5 * y_{\max }$ above the interface. This ensures that points in the trough-to-crest region are sampled at the same relative height above the interface, ensuring a more physical phase-averaged value in this region. This coordinate system is similar to the coordinate system applied by Vollestad et al. (2019a), evaluating the phase-averaged properties of airflow above waves.

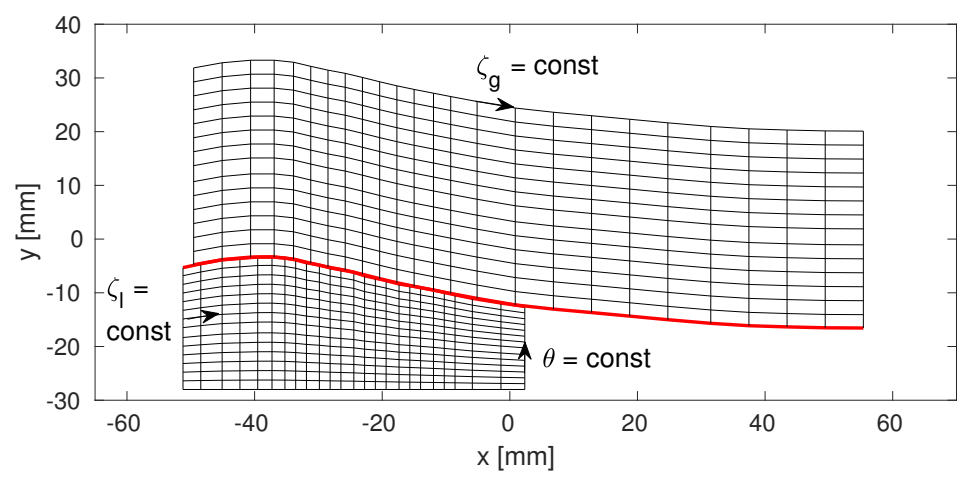

Figure 14: Example coordinate system. Only a small fraction of the gridlines included. Red line: interface.

The horizontal and vertical velocity components of breaking and non-breaking waves are sampled individually, and averaged at constant $(\theta, \zeta)$-coordinates to obtain the phase-averaged velocities $\langle u\rangle$ and $\langle v\rangle$. When performing the averaging, the average interface elevation (based on the LFV camera) is also evaluated. When presenting the phase-averaged results the $(\theta, \zeta)$-coordinates are transformed into $(\theta, y)$ - or $(x, y)$-coordinates (depending on the application) based on the mean interface. 


\section{References}

Ayati, A. A., Kolaas, J., Jensen, A. \& Johnson, G. W. 2014 A PIV investigation of stratified gas-liquid flow in a horizontal pipe. International Journal of Multiphase Flow 61, 129-143.

Ayati, A. A., Kolans, J., Jensen, A. \& Johnson, G. W. 2015 Combined simultaneous twophase PIV and interface elevation measurements in stratified gas/liquid pipe flow. International Journal of Multiphase Flow 74, 45-58.

Ayati, A. A., Kolahs, J., Jensen, A. \& Johnson, G. W. 2016 The effect of interfacial waves on the turbulence structure of stratified air/water pipe flow. International Journal of Multiphase Flow 78, 104-116.

BABAnin, A. 2011 Breaking and dissipation of ocean surface waves. Cambridge University Press.

BAnner, M. L. 1990 The influence of wave breaking on the surface pressure distribution in windwave interactions. Journal of Fluid Mechanics 211, 463-495.

Banner, M. L. \& Melville, W. K. 1976 On the separation of air flow over water waves. Journal of Fluid Mechanics $7 \mathbf{7}$ (04), 825-842.

Banner, M. L. \& Peirson, W. L. 1998 Tangential stress beneath wind-driven air-water interfaces. Journal of Fluid Mechanics 364, 115-145.

Belcher, S. E. \& Hunt, J. C. R. 1993 Turbulent shear flow over slowly moving waves. Journal of Fluid Mechanics 251, 109148.

Belcher, S. E. \& Hunt, J. C. R. 1998 Turbulent flow over hills and waves. Annual Review of Fluid Mechanics 30 (1), 507-538.

BIRVALSKI, M. 2015 Experiments in stratified gas-liquid pipe flow. TU Delft, Delft University of Technology.

Birvalski, M, Tummers, M. J., Delfos, R. \& Henkes, R. A. W. M. 2015 Laminar-turbulent transition and wave-turbulence interaction in stratified horizontal two-phase pipe flow. Journal of Fluid Mechanics 780, 439-456.

Birvalski, M., Tummers, M. J. \& Henkes, R. A. W. M. 2016 Measurements of gravity and gravity-capillary waves in horizontal gas-liquid pipe flow using PIV in both phases. International Journal of Multiphase Flow 87, 102 - 113.

Buckley, M. P. \& Veron, F. 2016 Structure of the airflow above surface waves. Journal of Physical Oceanography 46 (5), 1377-1397.

Gent, P. R. \& TAYlor, P. A. 1977 A note on separation over short wind waves. Boundary-Layer Meteorology 11 (1), 65-87.

Jessup, A. T., ZAppa, C. J. \& YeH, H. 1997 Defining and quantifying microscale wave breaking with infrared imagery. Journal of Geophysical Research: Oceans 102 (C10), 23145-23153.

KAWAI, S. 1981 Visualization of airflow separation over wind-wave crests under moderate wind. Boundary-Layer Meteorology 21 (1), 93-104.

KAWAI, S. 1982 Structure of air flow separation over wind wave crests. Boundary-Layer Meteorology $23(4), 503-521$. 
Kinara, N., Hanazaki, H., Mizuya, T. \& Ueda, H. 2007 Relationship between airflow at the critical height and momentum transfer to the traveling waves. Physics of Fluids 19 (1), 015102.

Kudryavtsev, V. N. \& Makin, V. K. 2001 The impact of air-flow separation on the drag of the sea surface. Boundary-layer meteorology 98 (1), 155-171.

MaAt, N. \& MAKin, V. K. 1992 Numerical simulation of air flow over breaking waves. BoundaryLayer Meteorology 60 (1-2), 77-93.

PhILliPs, O. M. 1958 The equilibrium range in the spectrum of wind-generated waves. Journal of Fluid Mechanics 4 (4), 426-434.

Phillips, O. M. 1985 Spectral and statistical properties of the equilibrium range in wind-generated gravity waves. Journal of Fluid Mechanics 156, 505-531.

Raffel, M., Willert, C. E., Scarano, F., Kähler, C. J., Wereley, S. T. \& Kompenhans, J. 2018 Particle image velocimetry: a practical guide. Springer.

Reul, N., Branger, H. \& Giovanangeli, J.-P. 2008 Air flow structure over short-gravity breaking water waves. Boundary-layer meteorology 126 (3), 477-505.

Siddiqui, K. \& Loewen, M. R. 2010 Phase-averaged flow properties beneath microscale breaking waves. Boundary-Layer Meteorology 134 (3), 499-523.

Siddiqui, M. H. K. \& Loewen, M. R. 2006 Detecting microscale breaking waves. Measurement Science and Technology 17 (4), 771.

Siddiqui, M. H. K. \& Loewen, M. R. 2007 Characteristics of the wind drift layer and microscale breaking waves. Journal of Fluid Mechanics 573, 417-456.

Smith, L., Kolaas, J., Jensen, A. \& Sveen, K. 2018 Investigation of surface structures in two phase wavy pipe flow by utilizing x-ray tomography. International Journal of Multiphase Flow 107, 246-255.

Strand, O. 1993 An experimental investigation of stratified two-phase flow in horizontal pipes. Dr. Scient. Thesis, University of Oslo. Oslo, Norway .

Sullivan, P. P., Banner, M. L., Morison, R. P. \& Peirson, W. L. 2018 a Impacts of wave age on turbulent flow and drag of steep waves. Procedia IUTAM 26, 174-183.

Sullivan, P. P., Banner, M. L., Morison, R. P. \& Peirson, W. L. $2018 b$ Turbulent flow over steep steady and unsteady waves under strong wind forcing. Journal of Physical Oceanography 48 (1), 3-27.

Tian, Z., Perlin, M. \& Choi, W. 2010 Observation of the occurrence of air flow separation over water waves. In ASME 2010 29th International Conference on Ocean, Offshore and Arctic Engineering, pp. 333-341. American Society of Mechanical Engineers.

Tulin, M. P. \& LAndrini, M. 2001 Breaking waves in the ocean and around ships. In TwentyThird Symposium on Naval HydrodynamicsOffice of Naval ResearchBassin d'Essais des CarenesNational Research Council.

Tzotzi, C. \& Andritsos, N. 2013 Interfacial shear stress in wavy stratified gas-liquid flow in horizontal pipes. International Journal of Multiphase Flow 54, 43 - 54. 
Ullmann, A. \& Brauner, N. 2006 Closure relations for two-fluid models for two-phase stratified smooth and stratified wavy flows. International journal of multiphase flow 32 (1), 82-105.

Veron, F., Saxena, G. \& Misra, S. K. 2007 Measurements of the viscous tangential stress in the airflow above wind waves. Geophysical Research Letters 34 (19).

Vollestad, P., Ayati, A. A., Angheluta, L., LaCasce, J. H. \& Jensen, A. 2019 a Ex690 perimental investigation of airflow above waves in a horizontal pipe. International Journal of Multiphase Flow 110, 37-49.

Vollestad, P., Ayati, A. A. \& Jensen, A. $2019 b$ Microscale wave breaking in stratified air-water pipe flow. Physics of Fluids 31 (3), 032101.

Weissman, M. A. 1986 Observations and measurements of air flow over water waves. In Wave dynamics and radio probing of the ocean surface, pp. 335-352. Springer.

YAng, Z., Deng, B.-Q. \& Shen, L. 2018 Direct numerical simulation of wind turbulence over breaking waves. Journal of Fluid Mechanics 850, 120-155. 